\title{
Circuit
}

Musiques contemporaines

CIRCUIT

\section{Regard sur quatre créateurs}

Jean Lesage, Subversive Composer ; Harvest what one sows.

The music of Sean Ferguson ; Michael Oesterle or The music of abstract ritual ; James Harley, A wandering Canadian

\section{Marc Couroux}

Volume 10, numéro 1, 1999

Québec : génération fin de siècle

URI : https://id.erudit.org/iderudit/004656ar

DOI : https://doi.org/10.7202/004656ar

Aller au sommaire du numéro

Éditeur(s)

Les Presses de l'Université de Montréal

ISSN

1183-1693 (imprimé)

1488-9692 (numérique)

Découvrir la revue

Citer cet article

Couroux, M. (1999). Regard sur quatre créateurs. Circuit, 10(1), 17-40.

https://doi.org/10.7202/004656ar
Résumé de l'article

À travers ces quatre textes, le pianiste Marc Couroux présente un portrait d'autant de créateurs chez qui, selon lui, la notion d'identités canadienne et québécoise est primordiale et intégrée à l'acte de création. Il situe les quatre compositeurs par rapport aux enjeux historiques de la création québécoise, tout en soulignant les particularités de l'esthétique de chacun d'entre eux. 


\section{Regard sur quatre créateurs \\ Marc Couroux}

\section{Jean Lesage, compositeur subversif}

All art has now become completely a game by which man distracts himself. What is fascinating now is that it's going to become much more difficult for the artist, because he must really deepen the game to be any good at all.

\author{
(Bacon, 1993) ${ }^{1}$
}

Jean Lesage est un compositeur subversif né en 1958, soit deux années avant la révolution tranquille. De prime abord, sa musique procède de la grande tradition européenne, elle apparaît comme le résultat d'un mimétisme on ne peut plus déférent. Méfions-nous, car les choses ne sont peut-être pas si simples! Tout d'abord, arrêtons-nous à notre rapport, en tant que Nord-Américains, à l'autre, l'Européen. La quête de notre nature propre, où si l'on veut de la manière dont nous nous définissons, me semble se faire par la négative. Lesage, qui utilise également ce mode de définition, prouve que cette démarche est aussi authentique que le degré zéro du modernisme ${ }^{2}$.

C'est la gratuité de l'art, son insoumission aux déterminismes d'une époque qui lui donne sa force révolutionnaire, son pouvoir subversif.

$$
\text { (Lesage, 1992, p. 47) }
$$

Les caractéristiques secondaires du discours de Lesage - mélodie, harmonie, rhétorique - se modèlent sur la tradition, sont issues des canons de la musique classique occidentale. La musique de Lesage posséderait donc tous les signes constitutifs qui permettent à l'auditeur-mélomane de la saisir immédiatement. Cependant, la continuité formelle orthodoxe, qui donnerait un sens à tous les autres paramètres, est manifestement absente. C'est pour cette raison que certains
1. Toutes les citations fournies par Marc Couroux ont été exceptionnellement reproduites dans la langue originale. (NDLR)

2. Voir l'article de Lesage intitulé "Non Serviam " in Circuit, vol. III, n 1 (1992) où il défendait de manière on ne peut plus éloquente le postmodernisme, en réponse aux propos sur l'avenir de la musique tenus par Pierre Boulez, porte-étendard de la modernité. 
auditeurs sous-évaluent la musique de Lesage, ils la jugent boiteuse ou croient qu'elle est le fruit de l'inexpérience, parce que ses éléments constitutifs impliquent un cheminement formel qui ne viendra jamais. Tout semble déséquilibré, incomplet. Cette dissociation entre structure et contenu est au centre du projet subversif de Jean Lesage. L'éternel débat quant à la capacité de l'auditeur à séparer contenu et contenant revêt ici une réalité quasi mythique. C'est précisément cette caractéristique qui donne à la musique de Lesage tout son poids sémantique.

L'œuvre que Lesage propose à son auditeur est, à un deuxième degré, particulièrement subversive. II la destine au mélomane amateur de musique classique, à celui qui possède une connaissance assez étendue du répertoire traditionnel. Lesage bâtit une relation de confiance avec cet auditeur tout en menant sournoisement une guerre souterraine contre les acquis culturels de ce dernier.

Half my painting activity is disrupting what I can do with ease.

$$
\text { (Bacon, 1993, p. 91) }
$$

Cette pensée formelle particulière et propre à Jean Lesage frappe par la force de ses implications. L'auditeur est amené insidieusement à repenser la fonction de chaque geste compositionnel. Par exemple, prenons la toute première mesure des Sensations confuses (1994) où une cadence classique sert de point de départ; cet archétype musical qui annonce la fin devient ici signe de la continuité, de la poursuite. Le geste familier ne laisse toutefois pas entrevoir sa finalité; ces fragments pourraient tous provenir en principe d'une seule pièce, éclatée en mille morceaux. Le "refrain» de La mémoire équivoque (1996), œuvre pour violon et piano, comporte une idée autonome par mesure (cf. exemple 1). Ces idées, complètement interchangeables, apparaissent dans un ordre différent à chaque reprise du refrain. 
Exemple 1, La mémoire équivoque, pour violon et piano, mesures 262-265.
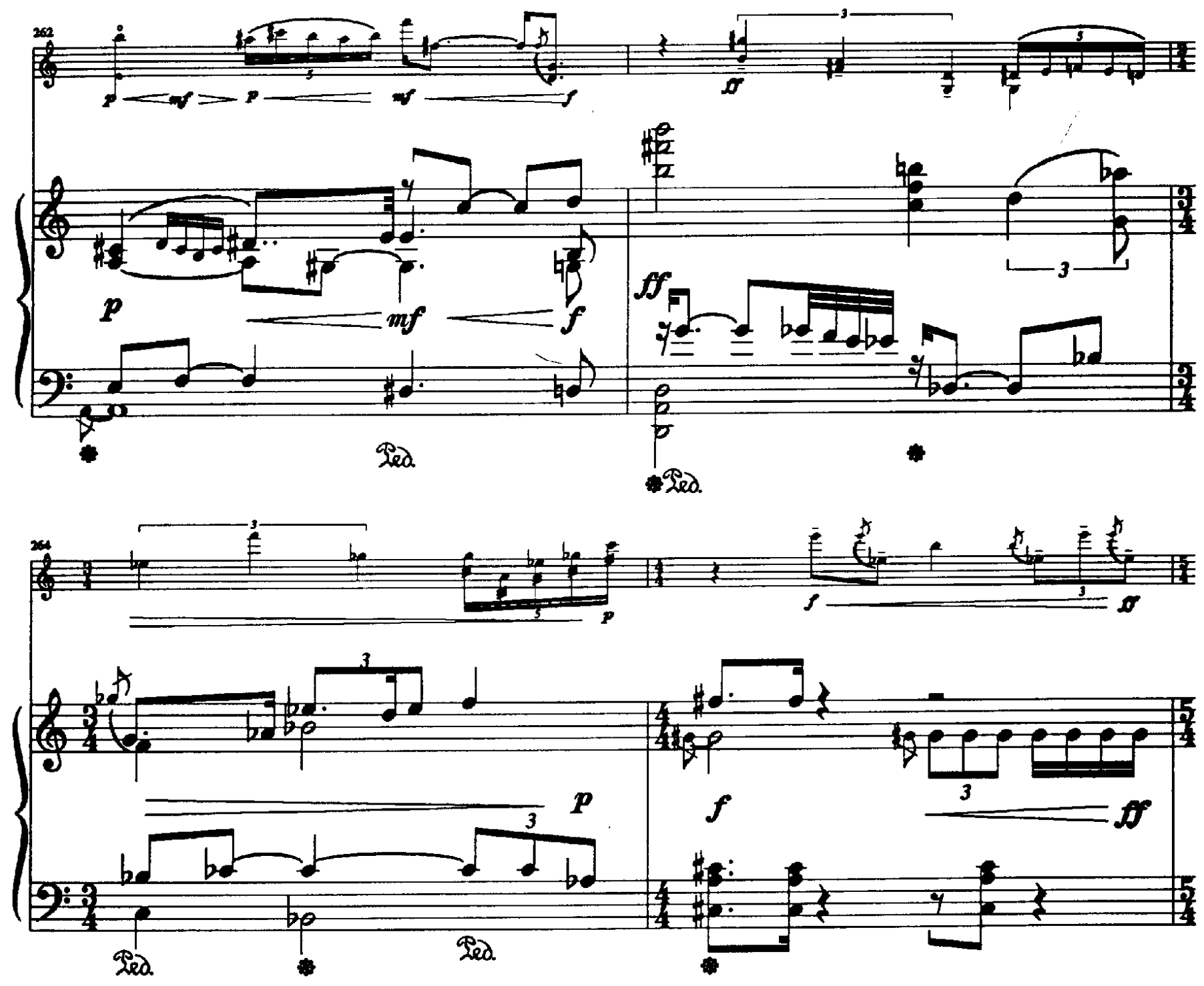


\title{
Ars tragica ou entreprise ludique?
}

Humpty Dumpty sat on a wall

Humpty Dumpty had a great fall

All the king's horses and all the king's men

Couldn't put Humpty Dumpty back together again

\author{
(Traditionnel)
}

Le discours, en perpétuelle rupture, nous renvoie à l'œuvre de l'américain John Zorn, qui se démarque, entre autres, par l'utilisation abondante de la méthode de juxtaposition rapide, caractéristique des dessins animés et du "zapping ". L'esthétique de Zorn reflète fidèlement la réduction de la capacité d'attention de l'Américain moyen. Toutefois, Lesage ne fait pas de rapiéçage éclectique, sa musique possède une cohérence stylistique bien à elle. Le compositeur s'inspire de l'Empfindsamkeit ${ }^{3}$, mouvement philosophique et esthétique du XVIII siècle, dans lequel s'inscrivait notamment C. P. E. Bach. On y trouve "une représentation musicale d'impressions, sentiments et passions souvent contradictoires, de brusques variations de climats, une succession rapide d'idées musicales antagonistes» (Lesage, 1997).

Does one know why very often, or nearly always, the accidental images are the most real ? Perhaps they've not been tempered with by the concious brain and therefor come across in a much more raw and real sense than something which as been tampered with by consciousness.

(Bacon, 1993, p. 176)

Inspiré par le langage des rêves, Lesage rejoint une réalité beaucoup plus culturelle qu'on ne pourrait le croire. Sigmund Freud divisait la psyché en trois parties: id, ego et superego. Ce dernier représente la conscience de l'humain, laquelle gouverne son comportement social, il est donc chargé d'interdits. Dans le rêve, le superego est radicalement marginalisé, cédant toute la place à l'id, l'instinct primaire indépendant de toute moralité. L'humain peut ainsi, lorsqu'il rêve, transgresser tous les codes interdits en temps éveillé.

L'œuvre pour piano Fantasia Stravagante (1997) marque un tournant important dans l'esthétique de Lesage. Plutôt que de procéder par juxtaposition externe d'éléments, Lesage opère une chirurgie à l'intérieur du matériau, altérant le code génétique, faisant surgir des chimères - dans la mythologie grecque, chimera était un monstre construit de parties incongrues. Beaucoup plus qu'un simple "trans-stylisme», le compositeur propose une véritable personnification des motifs, un "anthropomorphisme» cauchemardesque. Lesage croise les "gènes» de Guillaume de Machaut avec ceux d'Anton Webern, ceux de Claude Debussy avec ceux de Pérotin, pour produire des hybrides stylistiques nouveaux. A 
plusieurs reprises, I'id explose en une furie de notes, frôlant la folie utopique d'un Michel-Georges Brégent (1948-1993) et de son id qu'il censurait rarement. Ces passages, jusqu'à présent rares dans l'œuvre toujours astucieusement menée de Lesage, se fondent sur une série d'improvisations qui, bien que le travail se soit échelonné sur une année, furent toutes conçues à partir d'un même matériau. Lesage agit en archéologue, tentant de découvrir une logique secrète, faisant l'expérience de l'influence corrosive du temps sur la perception d'un matériau.

I believe that realism has to be continuously reinvented [...] the need to make changes in reality, which become lies that are truer than the literal truth.

$$
\text { (Bacon, 1993, p. 172) }
$$

Lesage soumet la déconstruction - et la reconstruction - de la musique ancienne à une alchimie toute personnelle. Sans se réfugier dans l'esthétique baroque, faute d'inspiration, Lesage veut plutôt faire revivre l'intensité de cette période à travers un langage actuel. II veut canaliser l'intensité du geste et de la rhétorique, agir sur le système nerveux de l'auditeur, grâce justement à l'intensité de ce geste et de cette rhétorique, comme Francis Bacon l'avait fait dans ses portraits déformés. À travers les distorsions rythmiques et harmoniques, Lesage réactualise ce qui a été banalisé par un langage tonal assimilé et répandu.

Mais pourquoi donc ces obsessions? pour les rêves, pour un passé essentiellement européen qui n'est pas le nôtre? Pourquoi se sentir interpellé?

Au Québec, dans les années quarante et cinquante, il me semble que le superego eut un poids grotesque, disproportionné, incarné par Duplessis et par un nationalisme religieux étroit ${ }^{4}$ qui étouffait toute pulsion créatrice jugée malsaine. En 1960, le premier ministre du Québec, Jean Lesage, présida à la Révolution tranquille, cette révolution culturelle qui fit entrer le Québec dans une ère plus libérale, où la religion perdit de son poids. Toutefois, et en dépit du fait que le clergé ait été évincé du pouvoir, il n'était guère possible que les Québécois se dotent immédiatement d'une culture propre, en raison du sous-développement de leurs réflexes culturels. Dans ce gouffre impitoyable, les compositeurs québécois se sont ralliés à la tradition européenne — une vraie tradition semblait-il —, pour combler ce vide apparent, qu'était leur propre héritage artistique.

Le compositeur Jean Lesage a reçu une formation traditionnelle de maîtres qui, comme tant d'autres musiciens issus du Conservatoire de musique, restaient attachés aux modèles européens. Lesage utilise toutefois son éducation "européenne » à son avantage, il garde une distance critique, se sert de signaux européens repérables, complètement assimilés, pour inciter à une contre-révolution ${ }^{5}$. On est bien loin d'une obéissance aveugle. On pense plutôt à une variation pervertie sur le thème, omniprésent, du Nord-Américain qui se compare sans cesse à l'autre, l'Européen.

Remarquons toutefois dans la musique de Lesage une atmosphère qui n'est pas loin de la tristesse, d'un certain regret, d'un mélodrame curieux lle spectre de
4. Qui glorifiait la ruralité.

5. Contre le sens et les référents culturels des symboles classiques. 
Mahler n'est jamais loin). Le discours en perpétuelles terminaisons des Sensations confuses contribue sans doute à ce sentiment. Malgré le postmodernisme avoué de Lesage, on frôle parfois - dangereusement même - une expression au premier degré, sans distanciation. Par ailleurs, il est intriguant de constater la présence, œuvre après œuvre, d'un signal que Lesage affectionne, une oscillation plaintive entre deux notes, une "mélodicité " rituelle, aussi caractéristique de la musique de Claude Vivier. Lesage rend-il un hommage inconscient à l'orphelin qui a su tendre un miroir à la société québécoise, sans pour autant lui proposer des solutions? À travers sa grande sensibilité et sa fine intelligence, Jean Lesage pose un regard nouveau par lequel, dorénavant, il parlera plus éloquemment de nous que de l'autre.

BACON, F. et Sylvester, D. (1993), Interviews with Francis Bacon, London, Thames \& Hudson.

FREUD, S. (1933), The Complete Introductory Lectures on Psychoanalysis, New York, W.W. Norton.

Latham, H. (1964), Mother Goose in French, New York, Thomas Y. Crowell Co.

LESAGE, J, (1992), "Non serviam», Circuit, revue nord-américaine de musique du XXe siècle, vol. III, no 1, p. 47-49.

LESAGE, J. (1997), Éléments de poétique musicale (inédit). 


\title{
Récolter ce que l'on sème. La musique de Sean Ferguson
}

If your language refutes your physical reality, then your problems are serious.

\author{
(Saul, 1997, p. 42)
}

Depuis une dizaine d'années, Sean Ferguson mène une réflexion approfondie sur l'existence même du compositeur canadien, existence qu'il juge plutôt précaire, et cela pour deux raisons. La première tient à la conjoncture culturelle, le compositeur de "musique de concert» vit dans un monde qui ne lui accorde que peu ou pas de place. La seconde raison est d'un tout autre ordre, celui du cheminement personnel, puisque le compositeur canadien est à la recherche d'une musique propre - typiquement canadienne si l'on veut — et non d'une musique qui se contenterait de calquer les tendances européennes.

Ferguson peut être considéré comme un héritier de R. Murray Schafer, qui amorça une réflexion sur l'identité musicale canadienne il y a près de trente ans et que l'on peut sans doute considérer comme le pendant musical de John Ralston Saul, philosophe canadien qui voit la nation canadienne comme une entité éminemment complexe (Saul, 1997, p. 9) et qui défend l'idée d'une culture canadienne très différente de celle des pays d'Europe.

Ainsi, la musique canadienne ne peut, selon Ferguson, être monolithique. Une telle conception, unitaire et globalisante, semblable en cela à certaines idéologies européennes, serait une distorsion démagogique et résulterait d'une insécurité chronique face aux Européens. De plus, elle ne pourrait s'appuyer que sur l'exclusion et la répression de ceux qui n'adhéreraient pas à cette fiction uniformisée.

"Culture," they explained, "you have none. Where you have a log house we have palaces. Where you have an axe we have grand pianos. Where you have a bog we have heated swimming pools. For this we do not blame you. Great art is not kept in a refrigerator. You need to mediterraneanize your existence. You also need people. Your cities are too small, too out of touch."

(Schafer, 1971)

There's a wonderful cliché, that a nation is great only as long as it has a frontier. We've got that frontier and this does something to the Canadian caracter, it means we've got a kind of civilization that does not conform to the rest of North America. Here is a place where non-conformism can live and flourish. 
To accept our reality - the myth of complexity - is to live out of step with most other nations. It is an act of non-conformity.

$$
\text { (Saul, 1997, p. 9) }
$$

Né en 1962 à Fort Vermilion, sur la frontière septentrionale de l'Alberta, l'expérience première de Sean Ferguson est faite d'un isolement presque total, il vivait loin de toute civilisation urbaine. Comme le personnage au début de la parabole Music in the Cold de Schafer, Ferguson a été formé et marqué par la brutalité de l'isolement canadien, le tourbillon des forces naturelles et le sentiment d'inaccessibilité que font éprouver les grandes distances. Ferguson met de l'avant l'idée d'un espace/temps canadien, unique en son genre:

Au Canada, nous pouvons circuler en voiture pendant une journée entière sans jamais sortir du pays, ni même de la province, voyageant parmi des gens qui partagent une même culture et une même langue. En Europe, en parcourant la même distance, nous pourrions avoir déjeuné, dîné et soupé dans trois pays différents, sans comprendre la langue d'aucun des habitants rencontrés'.

Ferguson s'étonne qu'un compositeur européen lui ait demandé, après l'écoute de l'une de ses œuvres: "Mais où est le matériau? » Ce compositeur n'avait pas compris que le matériau n'existe pas indépendamment de la forme "fergusonnienne». Indissociable du processus en mouvance perpétuelle, ce matériau n'est pas repérable à un moment précis dans le temps... il s'évapore aussitôł qu'il est saisi. En somme, le fait même de voyager devient le sujet, sans égard à un hypothétique point d'arrivée.

No matter how you cherish the wolf, he will always look back to the forest.

(Schafer, 1971)

Composé en 1997, le théâtre musical Son of Frankenstein est une œuvre "détour » qui servit de catalyseur et de thérapie durant une période de remise en question. Ferguson intégra Music in the Cold de Schafer, une œuvre à mi-chemin entre la légende et le tract social, à la célèbre histoire de Mary Shelley pour créer une mythologie dans laquelle Frankenstein, métaphore du compositeur canadien, délaissé et colonisé (le créateur de Frankenstein «force la terre de l'un pour nourrir l'autre de ses produits "), conduit à la métaphore de l'implantation inorganique d'idéologies européennes au Canada. Cette œuvre soulevait le problème de l'identité et de la raison d'être, ce à quoi il fallait trouver une solution.

An lrishman is never drunk so long as he can hold onto one blade of grass and not fall off the face of the earth.

\section{(Proverbe irlandais)}

Pendant un certain temps, me confia Sean Ferguson, je me suis laissé entraîné par l'idée que la communication dépend uniquement de la perception modelée par la culture de l'auditeur, c'està-dire par ses orientations, par ses goûts et par la mode. 
Mais cette attitude hyperrelativiste m'a troublé profondément et ce, presque jusqu'à la panne de créativité car j'avais l'impression qu'il n'existait pas de fondations solides et globales sur lesquelles le compositeur pourrait se reposer pour bâtir une œeuvre.

En musique, si le lien entre le symbole culturel et l'auditeur ne se fait pas, il y a très peu de communication véritable. Ainsi, au Canada, ce pays immense, une ceuvre construite uniquement à partir de signaux culturels risque de rejoindre un public incroyablement restreint. Je crois que la sensibilité à notre culture et à nos traditions, absolument indispensable mais loin d'être suffisante en soi, doit mener tôt ou tard à une expression personnelle. Bien qu'habité par sa réalité culturelle, un individu est fait d'un canevas beaucoup plus complexe que celle-ci. Cet état de fait nous mène à l'idée beaucoup plus globale de la communication entre compositeur et auditeur, finalement libérée des jugements de valeur culturels et locaux inhérents à une société humaine? 2 .

Ferguson a élaboré une conception de l'art qui, tout en s'écartant des modèles européens, semblent lui avoir été dictée par des tendances à l'hégémonie culturelle. Selon lui, "l'auditeur participe activement à la création d'une œuvre musicale». Cette œuvre est construite, en quelque sorte sculptée, dans l'esprit de l'auditeur et n'a aucune existence indépendante de celui-ci.

Ce ne sont pas les hauteurs et les durées qui constituent le véritable matériau compositionnel mais plutôt les facultés auditives et cognitives de l'auditeur. Les qualités objectives de l'œuvre sont étroitement liées aux qualités subjectives de la perception pdes auditeurs ${ }^{3}$.

Il n'existerait donc pas d'œuvre a priori, avant son assimilation par l'auditeur. Ce principe s'éloigne considérablement de certaines tendances esthétiques où le résultat sonore de l'œuvre est considéré en marge d'une conception abstraite et théorique de celle-ci.

À l'instar de ce que le critique d'art Roald Nasgaard a noté quant à "I'habileté du Nordique à réétablir le contact avec les sources premières de l'expérience» (Saul, 1997, p. 198), Ferguson a dégagé les embryons d'une identité nordique à travers une série d'œuvres telles que River Reflections (1 992), musique à processus dont la poésie tient à une exploration naissante de la perception de profondeur, ou Envolée-Marées (1994), deux pièces pour piano qui font appel, sans que le compositeur n'en ait eu véritablement conscience, à certaines notions psychoacoustiques $^{4}$ (cf. exemple 1). Depuis, le cheminement de Ferguson l'a amené à s'intéresser à ce domaine scientifique en plein essor.

Un des apports significatifs - et pourtant peu connu — du minimalisme est sans doute l'intégration de l'auditeur en tant que participant actif dans le processus créateur. Chaque auditeur peut véritablement construire sa propre version de l'œuvre, à partir de la perception qu'il en a. Les exégèses théoriques du minimalisme, aussi peu développées qu'elles soient, se sont concentrées principalement sur la forme, d'ailleurs peu développées en tant que processus graduel,
2. Ibid.

3. Ibid.

4. La psychoacoustique est l'analyse des relations entre les propriétés du son lforme d'onde, spectre, niveau, fréquence) et la façon dont ces sons sont perçus (volume, hauteur, timbre, saillance). (Parncutt, 1989) 
Exemple 1, Envolée-Marées, pour piano, mesures 87-98.
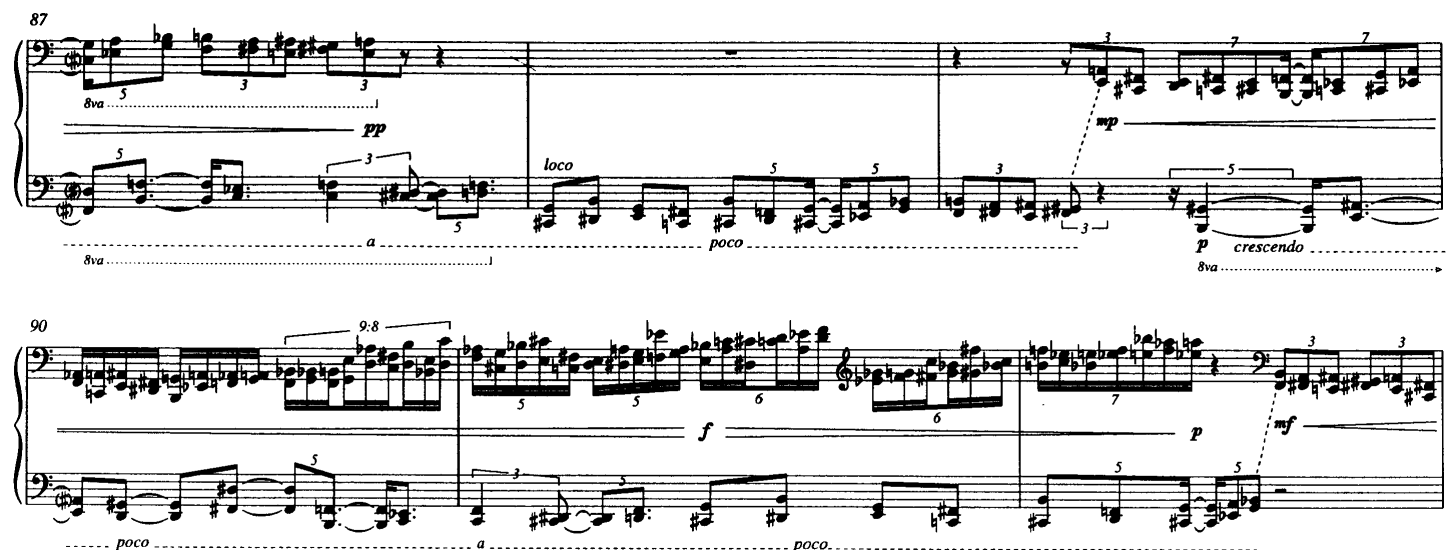
sva ...................................... loco
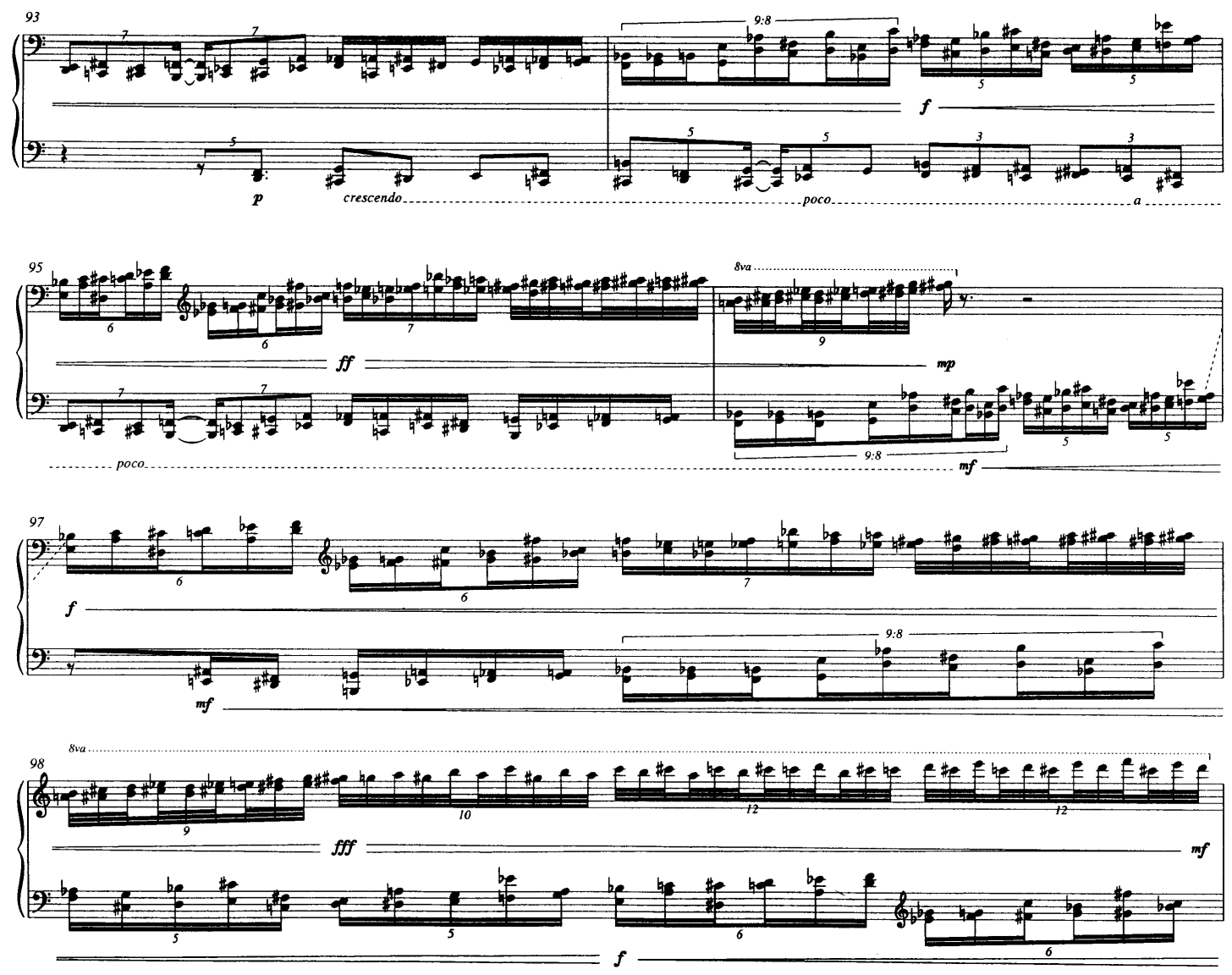
plutôt que sur les particularités psychoacoustiques résultantes. Une musique comme celle de Steve Reich dans les années 1970 (Drumming, Six Pianos) est radicale par sa volonté d'explorer directement la perception de l'auditeur sans arguments dramatico-expressifs. Ferguson explore ces idées, tout comme Glenn Gould qui voulait, en manipulant ses bandes après les séances d'enregistrement, abolir la perception acoustique unidimensionnelle du répertoire traditionnel, au profit d'une vision beaucoup plus «réaliste» de l'œuvre.

Au début des années 1980, la musique de John Rea explore à son tour les transpositions potentielles de l'illusion d'optique sur le plan sonore. Treppenmusik (1982) comporte en effet une section basée sur les Shepard tones, un principe psychoacoustique connu (ainsi qu'une métaphore des escaliers infinis de Maurits Escher). Cette œuvre eut une influence considérable sur Ferguson. La fascination pour la mécanique de la perception et le désir de l'utiliser comme matériau de base, un des éléments qui façonne l'œuvre de Ferguson, sont probablement aussi des traits typiquement nord-américains.

Even when all the cards are on the table and everyone hears what is gradually happening in a musical process, there are still enough mysteries to satisfy all. These mysteries are the impersonal, unintended, psycho-acoustics by-products of the intended process.

$$
\text { (Reich, 1968, p. 7) }
$$

Sean Ferguson se confie à nouveau :

J'ai grandi dans un village de 600 personnes, au sein d'une communauté majoritairement autochtone. J'étais membre d'une minorité visible, n'ayant virtuellement rien en commun avec la culture qui m'entourait. De plus, je n'avais pas de liens avec le monde extérieur; nous n'avions pas même de téléviseur. Quand j'étais jeune, les différences culturelles entre moi et mon entourage étaient évidentes. Aujourd'hui [à Montréal], ces différences sont plus subtiles mais néanmoins tout aussi réelles. Depuis que j'ai quitté mon village, je réalise que je n'ai jamais eu la chance de développer un sentiment très fort d'appartenance culturelle, peu importe où je me retrouve dans le monde. J'ai toujours eu le sentiment d'être un marginal.

Malgré les différences culturelles que je ressens et qui existent entre les sociétés à travers le monde, je crois sincèrement qu'il est possible de communiquer au-delà de ces frontières. Je ne pourrais pas composer si je n'avais pas cette croyance ${ }^{5}$.

Sean Ferguson s'inscrit ainsi dans une lignée de Canadiens, à laquelle appartiennent les peintres du groupe des Sept et les signataires de Refus global; lignées d'artistes manifestement destinées à repousser les barrières culturelles pour atteindre un degré de communication beaucoup plus universel entre l'art et le public. "Je suis convaincu, disait Paul-Émile Borduas, qu'où je me reconnais dans mon être le plus intime, le plus particulier, des millions d'autres pourront aussi se reconnaître. 》(Saul, 1997, p. 206)
5. Communication personnelle de Sean Ferguson à Marc Couroux. 
GoulD, G. (1967), The Idea of North (documentaire radiophonique), CBC Records PSCD 2003-3.

Parncutt, R. (1989), Harmony: A Psychoacoustical Approach, Berlin, Springer-Verlag.

RelCH, S. (1968), Music as a Gradual Process, Deutsche Gramophon DG 427 428-2.

SaUl, J. R. (1997), Reflections of a Siamese Twin, Toronto, Penguin.

SChafer, R. M. (1971), Music in the Cold, Peace River, Arcana Editions. 


\section{Michael Oesterle, ou la musique du rituel abstrait}

When you're outside a tradition, as every artist is today, one can only want to record one's own feelings about certain situations as closely to one's own nervous system as one possibly can.

(Bacon et Sylvester, 1993, p. 43)
La musique de Michael Oesterle comporte une forte dimension mythologique. $C^{\prime}$ est une musique rituelle abstraite, un retour du compositeur à une source résolument "préverbale» de l'expérience musicale, matérielle et magique, ce qui lui donne sa grande force communicative.

Né en 1968 à Ulm, dans le sud de l'Allemagne, Michael Oesterle vit au Canada depuis son adolescence. II reste toutefois lié inextricablement à la culture européenne, peu importe qu'il s'y reconnaisse ou non. La tradition européenne ${ }^{1}$ est un aimant puissant qui transforme, à court ou à long terme, celui qui se trouve dans son pôle d'attraction. Puisque Oesterle est européen, il ne peut éviter le faceà-face avec sa tradition, que l'issue en soit positive ou négative.

La musique d'Oesterle était peutêtre européenne à son origine, mais ses fragments ont depuis quitté le vieux continent, à la recherche de leur propre identité, voguant dans des contextes qui n'ont plus de relation explicite avec les structures de pensée européennes.

Le cinéaste russe Andrei Tarkovsky exerce une fascination révélatrice sur Oesterle. Pour Tarkovsky, l'icône religieuse, omniprésente dans ses films, symbolise la recherche obsessionnelle du salut, quasi inaccessible en cette ère profondément matérialiste. Les icônes sont des repères sur le chemin d'une foi à laquelle il est toujours difficile d'accéder. Dans la musique d'Oesterle, les icônes incarnent le sédiment d'une longue concentration, d'un processus de réflexion sur la musique. Elles sont les fruits d'une purification constante, n'admettant que les vérités distillées, que l'essence.

Still (1995), sa première œuvre marquante, met en relief la progression d'îlots iconiques de matériau à travers un cheminement sans destination. II n'y a pas de préalable structurel chez Oesterle, dont les formes sont développées au fur et à mesure de l'écriture. Luigi Nono, un compositeur marginal - qui s'est détaché esthétiquement de la scène européenne tout en en faisant partie physiquement -, a bien dit qu'il "n'y a pas de chemin, il n'y a que le cheminement ${ }^{2}$ ". (Balázs, 1987, p. 331
1. On entend par cette expression la totalité de la grande tradition européenne de musique dite sérieuse, de Pérotin à nos jours.

2. "No hay caminos, hay que caminar». Nono a fait sienne cette inscription ancienne lue sur un mur de Tolède, et a donné à une œuvre de 1989 le titre "Hay que caminar soñando". Traduction libre de Jean Lesage. 
Exemple 1, Reprise, mesures 1 à 14.

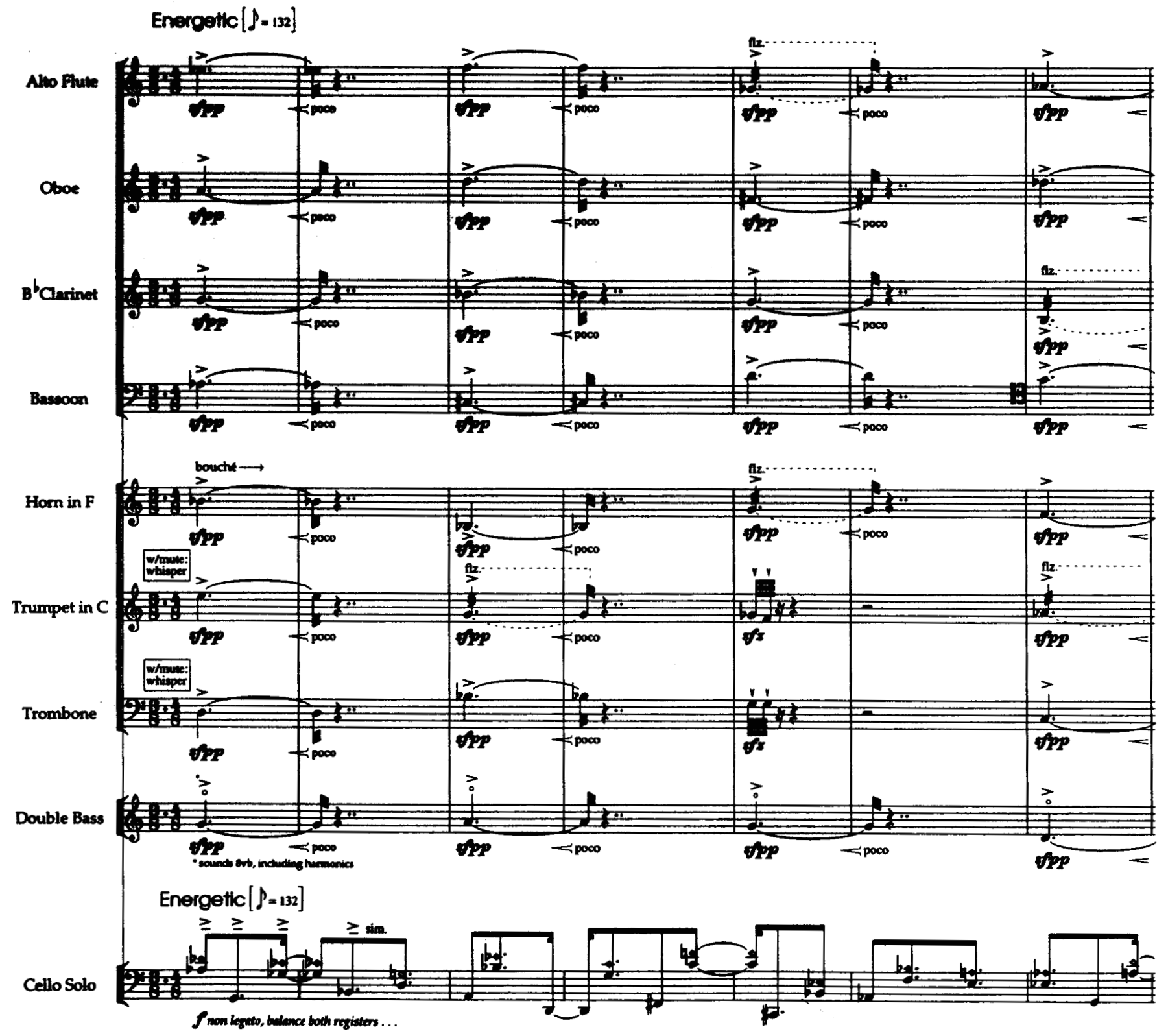

- michael oesterle (97) 
(8)

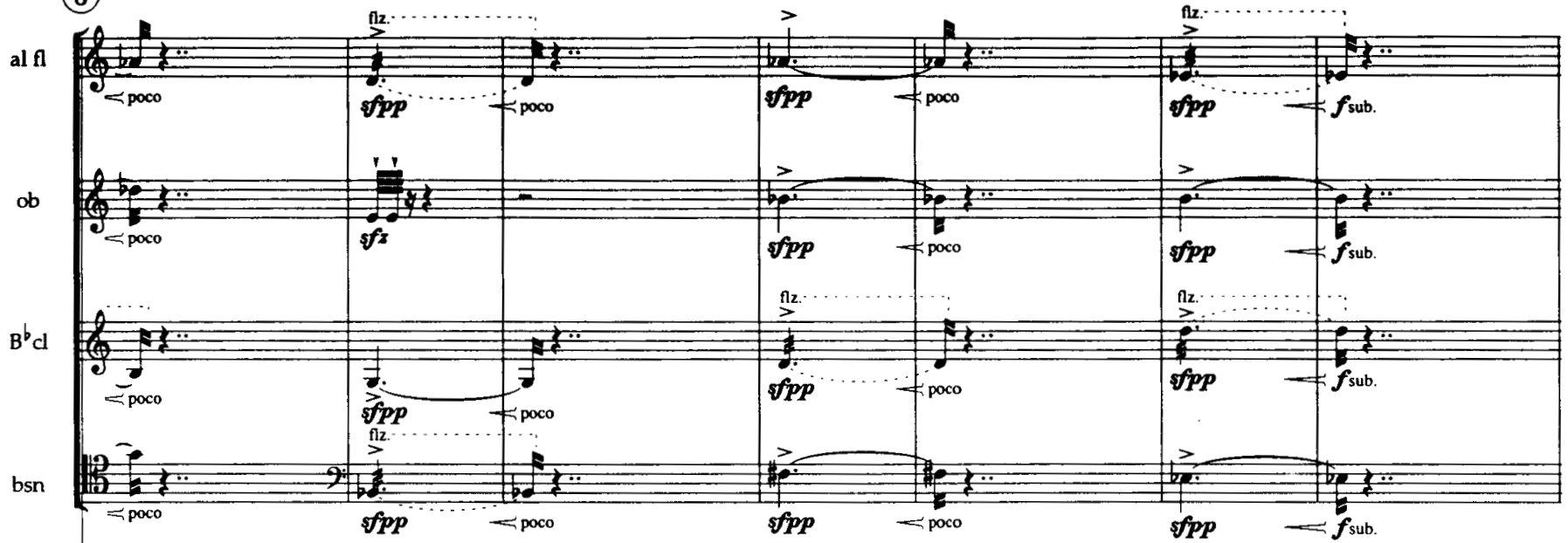

hm

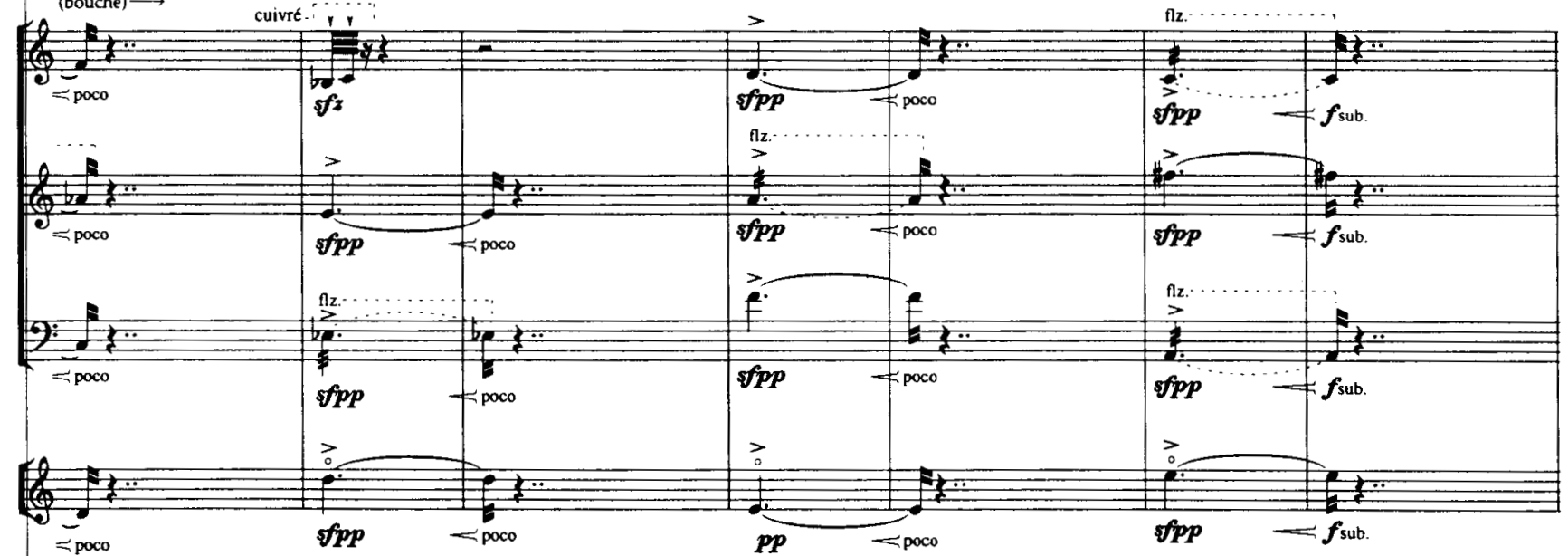

vc

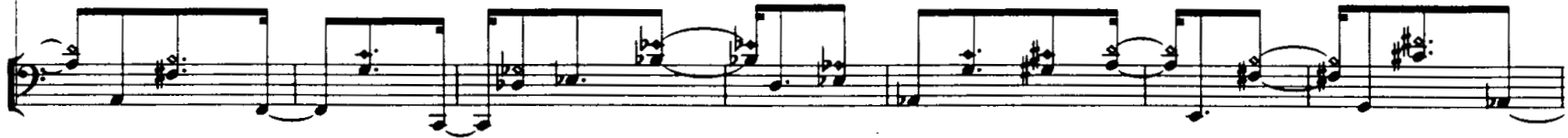




\section{Musica materialis}

Dans la musica materialis d'Oesterle, le matériau est le sujet même de l'œuvre. La distinction entre avant- et arrière-plans texturés est oblitérée. Oesterle crée son univers à partir de données tangibles, connues, expérimentées, perçues. II n'y a jamais rien qui ne soit signifiant. À partir du moment où un matériau devient aussi naturel que la respiration, on assiste à un élargissement quasi infini du réseau de possibilités. Oesterle renoue avec l'idée de construire un corpus d'œuvres sur un matériau on ne peut plus restreint. II y a ici identification totale entre le compositeur et son matériau, une relation parfois même exagérée, qui excède ses limites. Chaque œuvre est une tentative additionnelle de réduire le matériau à sa structure moléculaire de base. Elle s'inscrit dans une esthétique de la raréfaction. Son acharnement à atteindre un but, quels que soient les obstacles sur le parcours est remarquable. II s'agit là de traits tout à fait modernes et occidentaux, mais d'un modernisme non européen, non hiérarchique. On pourrait même voir dans cette optique une recherche empirique jungienne d'archétypes, ainsi que la possibilité de retrouver les sources fondamentales de l'expérience humaine. Une allégation forte certes, mais c'est peut-être pourquoi l'œuvre d'Oesterle a sur l'auditeur un impact qui va bien au-delà de celui d'une musique contemporaine normalisée ${ }^{3}$.

L'esthétique d'Oesterle, radicalement expérimentale, soulève plusieurs questions : est-ce qu'un matériau quelconque peut exister dans le vide, dénué de toute connotation ou de tout sens extramusical ? Est-ce que le matériau peut engendrer un sens en ne se référant qu'à lui-même, être en quelque sorte autosuffisant?

The new man, linked in a cosmic harmony that transcends time and space, will sensuously caress and mold and pattern every facet of the terrestrial artifact as if it were a work of art, and man himself will become an organic art form.

$$
\text { (Mcluhan, 1995, p. 268) }
$$

Oesterle renove avec une esthétique bien loin des courants européens actuels; il se tourne vers ce qu'on pourrait appeler l'animisme ${ }^{4}$. Caractéristique de l'art des pays nordiques, l'animisme est à l'opposé d'une structure orientée vers un but précis. C'est plutôt l'infusion d'une âme, d'une vie indépendante qui serait attribuée au matériau lui-même, qui est un trait animiste. On se souviendra de Morton Feldman, qui voulait accorder une "mort naturelle» à ses sons et à ses formes. II n'est pas surprenant qu'Oesterle ait adopté cette attitude animiste, justement parce qu'il est loin des "centres officiels» de musique contemporaine, où on remarque depuis longtemps, à peu d'exceptions près, un divorce entre l'art et la géographie. II est installé dans un pays où l'animisme est encore une réalité. Dans Reprise (1997), une série de sept accords semble vouloir se détacher des contraintes temporelles de l'œuvre, du contexte linguistique, pour germer ailleurs. Le matériau, on ne peut plus restreint, perdure dans l'esprit bien longtemps après que la musique soit terminée (cf. exemple 1).
3. De plus en plus, on remarque un conformisme stylistique étonnant dans la musique contemporaine (chez les maîtres autant que chez les apprentis), une pénurie de rhétoriques dépassant le canon traditionnel. L'avant-garde est bel et bien institutionnalisée et ce, à travers le monde entier.

4. Attitude, croyance, religion selon laquelle les animaux, les objets et les phénomènes naturels ont une âme. Définition tirée du dictionnaire informatique Antidote. 


\section{D'une insatisfaction}

En un sens plus large, la recherche d'Oesterle découle d'une insatisfaction visà-vis des systèmes jugés "historiquement valables», une idée parmi bien d'autres véhiculée par les postsériels. Le minimalisme, la musique spectrale, la nouvelle complexité, etc. sont tous des codes, qu'ils aient été à l'origine de mouvements révolutionnaires ou non. Ce déplacement paradigmatique est important et il s'ensuit, pour Oesterle comme pour plusieurs compositeurs de sa génération, un refus du principe de "l'art pour l'art», figé dans le formalisme et les cultes stylistiques.

Par l'intensité de son esthétique, Oesterle provoque une réflexion globale sur le matériau; mis en présence du seul noyau essentiel étendu sur une longue période, l'auditeur n'a d'autre choix que de se concentrer et de pénétrer dans la logique interne du son. Le champ d'exploration artistique paraît de nouveau illimité. Dissocié de la tradition européenne, le drame se joue à un niveau supérieur, l'infiniment petit prenant des proportions gigantesques. II suffit d'ajuster la «lentille», par exemple, sur le paramètre du tempo, pour intensifier le drame de façon efficace.

The art of the North is composed of tiny events magnified.

Those accustomed to fat events that don't matter,

Or to many events, miss the details.

To them the winter soundscape is 'silent' as snow is merely 'white'.

(Schafer, 1971)

Oesterle insuffle au matériau un mystère, il renoue avec son caractère organique et fébrile. En quête d'identité, Oesterle creuse d'une manière obsessionnelle vers le noyau. II s'engage dans une dialectique personnelle qui reflète à la fois ses origines européennes et le regard que l'Amérique du Nord porte sur cellesci. Le plus extraordinaire est qu'Oesterle réussisse à communiquer cet état esthétique sans avoir recours à la manipulation consciente des perceptions culturelles de son auditeur. La dialectique est implantée dans I'ADN du matériau et n'a besoin d'aucun langage critico-symbolique pour attirer notre attention ${ }^{5}$.

For the moment we are witnessing the decline of the spiritual while the material long ago developed into an organism with its own bloodstream, and became the basis of our lives, paralyzed and riddled with sclerosis. We have reached the point where the present has essentially merged with the future, in the sense that it contains all the preconditions for immanent disaster; we recognise this and yet we can do nothing to stop it happening... The point as been reached where we seem to have a fatal incapacity for mastering our material achievements in order to use them for our own good.

(Tarkovsky, 1986, p. 235)
5. Il est révélateur de comparer cette démarche à celle de Jean Lesage (voir précédemment dans ce numéro), qui est basée sur le détournement, sinon la perversion des symboles musico-culturels. 
Peut-être l'avenir n'est-il pas aussi sombre que Tarkovsky le décrit. Chose certaine, la musique de Michael Oesterle nous mène en un terrain dangereux, où il est fort possible de se perdre en un culte stérile du matériau, immobile et inflexible. Toutefois, l'intensité avec laquelle son matériau lutte pour s'imposer à nous, nous fait supposer que la musique d'Oesterle a la capacité de prendre sa place dans la marche globale vers un humanisme qui se définira hors de codes conformistes et de contextes historiques progressistes.

BACON, F. Et Sylvester, D. (1993), Interviews with Francis Bacon, London, Thames \& Hudson.

BAĹÁZ, I. (1987), "De l'actualité artistique de Luigi Nono», Contrechamps, Festival d'automne à Paris 1987, Paris.

Mcluhan, M. (1995), Essential Mcluhan, Concord, Anansi.

Schafer, R. M. (1971), Music in the Cold, Peace River, Arcana Editions.

Tarkovsky, A. (1986), Sculpting in Time, Austin, University of Texas Press. 


\section{James Harley, un Canadien errant}

Pendant longtemps, je me suis senti incapable d'écrire un texte sur la musique de James Harley. J'étais franchement déboussolé par la diversité de son œuvre, manifestement privée d'un style global, unificateur et rassurant. II semblait peu probable que le même compositeur ait été à l'origine du jazz-bop de l'Étude pour une Fête (1991), du récessif Kekula (1992), de l'intransigeant Kaleidarray (1994), du classique Neve Bilder (1991) et du folklorique Cuimnheachan Urramach (1996). Harley est un Canadien errant typique. Omnivore, ses champs d'intérêt ne peuvent être classés sous une rubrique esthétique étroite; la question d'un style unificateur potentiel ne se pose même pas. Harley est éclaté et complexe, comme son pays. R. Murray Schafer appartient aussi à cette "tradition canadienne de l'errance», une esthétique qui refuse toute catégorisation sommaire. Plutôt que d'évolver à partir d'une idéologie centriste, chaque œuvre de Harley se situe à l'intersection d'un réseau d'influences.

J'ai grandi à la campagne, pas à la ville, dans un endroit qui dominait le lac Kalamalka, le "lac de multiples couleurs" - c'est très juste - avec une vue de la vallée aux montagnes couvertes de neige, les Pics des Monashees. Le Canada est un pays de terroir, de vastes étendues, du Nord, un pays sauvage parsemé d'infimes fenêtres de civilisation. Le sentiment que j'aimerais exprimer en musique est identique à celui que je ressens sur le sommet d'une montagne, baignant dans une infinité de montagnes, d'arbres, de lacs, de neige... un rapport avec l'émerveillement, le mystère, la sainteté ?

Né en 1959 à Vernon en Colombie-Britannique, Harley vit tout jeune en 1. Communication personnelle de James Harley à Marc Couroux. étroite symbiose avec la nature, relation qui déterminera une grande partie de son esthétique.

The place is never the background... it is a leading character.

\section{(Saul, 1997)}

Peut-être qu'à force de refuser d'intégrer le monde naturel canadien à nos pratiques artistiques, refus imputable à un embarras tout à fait provincial, nous avons sacrifié l'aspect le plus typique de notre culture. L'œuvre de Harley met au premier plan une sensibilité à la dureté et à la rugosité de la nature canadienne. Sa vision n'a rien en commun avec un quelconque "pastoralisme», encore moins avec le courant néo-mystique de l'Europe de l'Est. Plutôt, Harley accepte le fait que la complexité de la nature exige une interprétation artistique plus imaginative qui dépasse la simple contemplation placide et surannée. Les récits de l'auteure américaine Annie Dillard, qui s'isola dans le nord-ovest américain, véhiculent aussi l'idée de la violence irréductible de la nature comme métaphore de la 
créativité. Par ailleurs, il est peu surprenant que l'une des premières biographies d'Edgard Varèse ait été rédigée par un Canadien, le poète et peintre québécois Fernand Ouellette; celui-ci a pu sentir dans l'austérité et le magma sonore varésien - qui cadrait très peu avec les courants européens - quelques affinités avec le paysage canadien.

Harley s'orienta pour sa part vers la théorie du chaos, une discipline alors en pleine éclosion, dont les principes dérivent d'une étude beaucoup plus globale des mécanismes naturels que ce que permettait auparavant l'hyperspécialisation. À partir de 1988, Harley développe Chaotics, un logiciel basé sur quelques-unes des idées fondamentales de cette théorie, en particulier les "attracteurs étranges », qu'il utilise pour générer des hauteurs et des rythmes.

La progression formelle des œuvres de Harley dénote un curieux parallèle avec le concept de rhizome, concept au centre de la pensée des philosophes français Gilles Deleuze et Félix Guattari, et qui prolonge l'esthétique naturelle:

À l'opposé d'une structure qui se définit par un ensemble de points et de positions... le rhizome n'est fait que de lignes. Le rhizome est une antigénéalogie. C'est une mémoire courte, ou une anti-mémoire... Le rhizome est un système acentré, non hiérarchique et non signifiant, sans général, sans mémoire organisatrice ou automate central, uniquement défini par une circulation d'états.

(Deleuze et Guattari, 1987, p. 21)

Étonnamment, ces propos rejoignent l'expérience canadienne, telle qu'analysée par John Ralston Saul: "La complexité (canadienne) [...] requiert une recherche d'équilibre entre les éléments, sans éradication ou domination, mais une lutte pour développer et préserver l'équilibre. »(Saul, 1997, p. 223) 2

2. Traduction libre de Marc Couroux.

Les œuvres de Harley habitent ce moyen terme mystérieux où il est difficile de discerner l'élément clé qui engendre le mécanisme global. Dans Octane-VX (1996), le riche réseau d'interrelations apparentes fait en sorte que l'auditeur ne peut opérer de quelconque hiérarchisation des éléments. Cet organisme affirme plutôt son caractère indivisible, chacune des lignes est indispensable, sans toutefois qu'aucune ne provienne d'un paradigme structurel originel. On est loin d'une temporalité dramatique. L'œuvre de Harley reflète assez fidèlement le pragmatisme de l'Américain William James. En effet, ce dernier défend une conception de la vie comme "expériences pures», "en attente à l'intérieur du flux de la vie». Uames, 1987) ${ }^{3}$

Ce sont les littératures américaine, et d'abord anglaise, qui ont manifesté ce sens rhizomatique, ont su se mouvoir entre les choses, instaurer une logique du ET, renverser l'ontologie, destituer le fondement, annuler fin et commencement. Ils ont su faire une pragmatique." 
Kaleidarray (Jazz III) pour clarinette, trompette, violon, piano et deux percussionnistes (1994), composé pour l'ensemble torontois Arraymusic, est une musique folklorique purement imaginaire qui fusionne des éléments provenant de traditions «jazzistiques» et orientales, traditions placées sur le même pied d'égalité dans l'esthétique éclatée de Harley. On remarque d'abord la liberté rythmique du trio clarinette-trompette-violon, asynchrone par rapport à la pulsation sous-jacente maintenue par les trois autres instrumentistes. Un drame "secret» se joue dans l'affrontement entre le cantus (les éléments mélodiques, discursifs) et le continuum (la pulsation régulière). Le cantus assume souvent un poids dramatique exagéré, tissant ses mélodies lyriques presque à l'insu du continuum. La mélodicité hétérophonique et la structure rythmique font penser au gamelan balinais (cf. exemple 1).

Exemple 1, Kaleidarray (Jazz III), mesures 58 à 67.

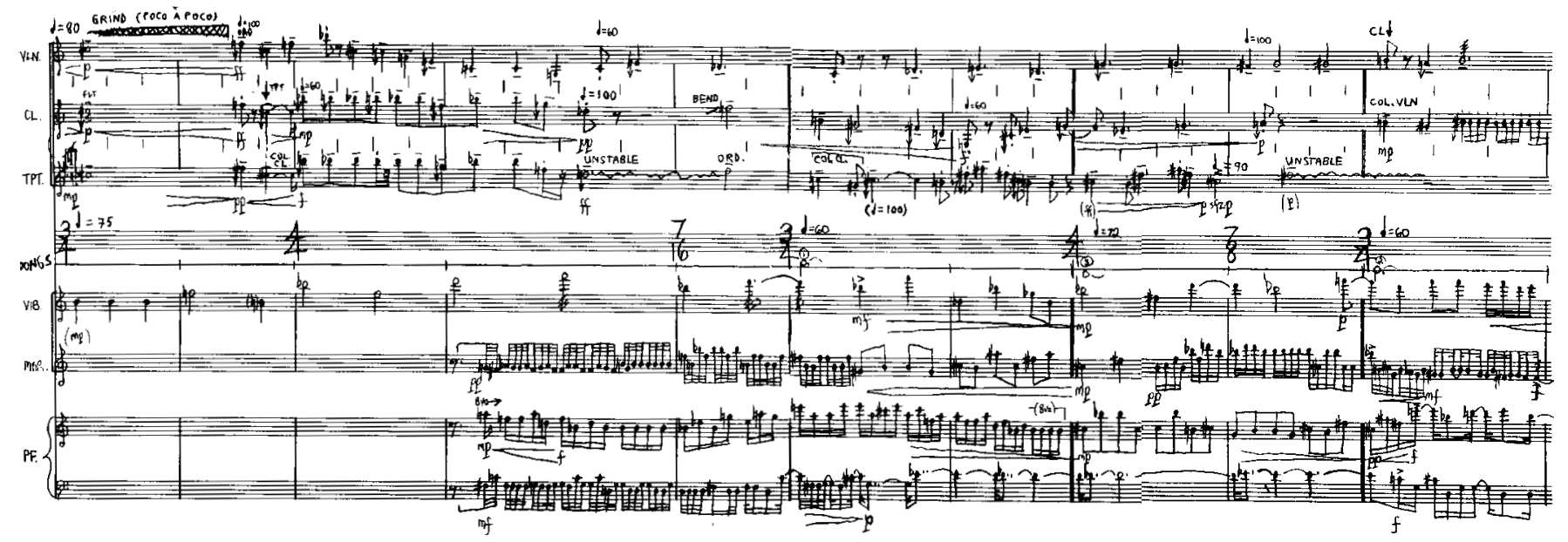

Une étrangeté volontaire émane du glissement sémiologique entre la placidité du continuum et l'instabilité du cantus. Certes, le syncrétisme ritualisé de l'Art Ensemble of Chicago a exercé ici son influence. Par la cœexistence des éléments hétérogènes, Harley force l'auditeur à redéfinir ce qui est ou non dramatique, intentionnel et non intentionnel. Comme chez Charles Ives, ces musiques coexistent par leur force brute, par une volonté d'anarchisme pur, et c'est justement ce frottement qui révèle l'essence de chacune d'elles, sans pour autant que le tout soit réuni sous forme de platitudes esthétiques globales. Kaleidarray est difficile d'accès, car aucune concession n'adoucit le propos irréductible: c'est un paysage canadien typique, irrationnel et intransigeant.

The Canadian sensibility is that of the edge, the unknown, the uncontrolled - place and art are the same thing [...] There is no separateness of men from the elements, no sense of a possible control.

(Saul, 1997, p. 205) 
La formation de musicien de jazz que Harley a reçu à la Western Washington University à Bellingham l'a sensibilisé à la tradition créatrice nord-américaine perpétuée par, entre autres, le pianiste Cecil Taylor, l'Art Ensemble of Chicago et Harry Partch, mais aussi par Jimi Hendrix et sa fameuse version du Star-Spangled Banner. L'aura "décorporalisée » de ces musiciens, dont le jeu très physique semble, paradoxalement, échapper aux contraintes matérielles, a stimulé Harley, déjà séduit par les récits de Carlos Castaneda et son art de "voir " le monde dans toute sa réalité magique, et parfois violente, sans influence de l'intellect. L'errant Glenn Gould était obsédé par ce même phénomène, qu'il nommait extase, provoquée par une brutalité de l'expérience qui résulte en une suspension du temps et un "positionnement en dehors de soi » (Payzant, 1992, p. 63).

En 1995, isolé dans la nature sauvage de Banff, je recevais quotidiennement par la poste les pages de Flung loose into the stars (1995), une œuvre pour piano de James Harley. Cette ouvre fut au départ une recherche d'analogies, notées, avec la pyrotechnie improvisée de Cecil Taylor, mais elle se développa loin de ces sentiers. Dans son sens le plus large, l'œuvre est plutôt une représentation on ne peut plus fidèle d'une vie en périphérie de la civilisation, de l'inconnu. Le contrepoint à huit voix est le prétexte à une séparation extatique entre le corps et le son, pour les recombiner sur un plan plus élevé. Flung loose into the stars ne s'inscrit pas dans la conception foncièrement européenne du virtuose qui exhibe ses talents devant une foule. Comme on le sait, Glenn Gould abandonna tôt dans sa carrière cette poursuite " hérö̈que " pour explorer l'isolement créatif, cette lutte solitaire pour renouer avec «l'énorme possibilité créative que représente le côté physique du pays " (Gould, 1967) ${ }^{4}$.

For Canadians, whatever their language or politics, the acceptance of complexity has meant the acceptance of a perpetually incomplete experiment.

$$
\text { (Saul, 1997, p. 13) }
$$

James Harley est l'un de ceux qui affrontent quotidiennement l'idée d'être canadien, ce qui demande beaucoup plus de courage et d'imagination qu'un simple cri de ralliement stylistique nationaliste. À l'aise au sein d'une pléthore de mythologies canadiennes, Harley vit au carrefour du village global de Marshall McLuhan, ce pionnier qui croyait profondément "en l'homme et son potentiel de grandir et d'apprendre, d'explorer la profondeur de son être et de découvrir les mélodies secrètes qui orchestrent l'univers» (McLuhan, 1995, p. 268). James Harley défend au plus haut point la grande tradition créatrice canadienne, enracinée dans un monde naturel qui est une métaphore de l'irréductible complexité du Canada et, par prolongation, de l'humanité tout entière. 
Deleuze, G. et F. Guattari (1987), A Thousand Plateaus, Minnesota, University of Minnesota Press.

Gould, G. (1967), The Idea of North (documentaire radiophonique), CBC Records, PSCD 2003-3.

JAMES, W. (1987), Writings 1902-10, New York, Library of America.

Mcluhan, M. (1995), Essential Mcluhan, Concord, Anansi.

Payzant, G. (1983), Glenn Gould: Music and Mind, Toronto, Key Porter.

SaUl, J. R. (1997), Reflections of a Siamese Twin, Toronto, Penguin. 


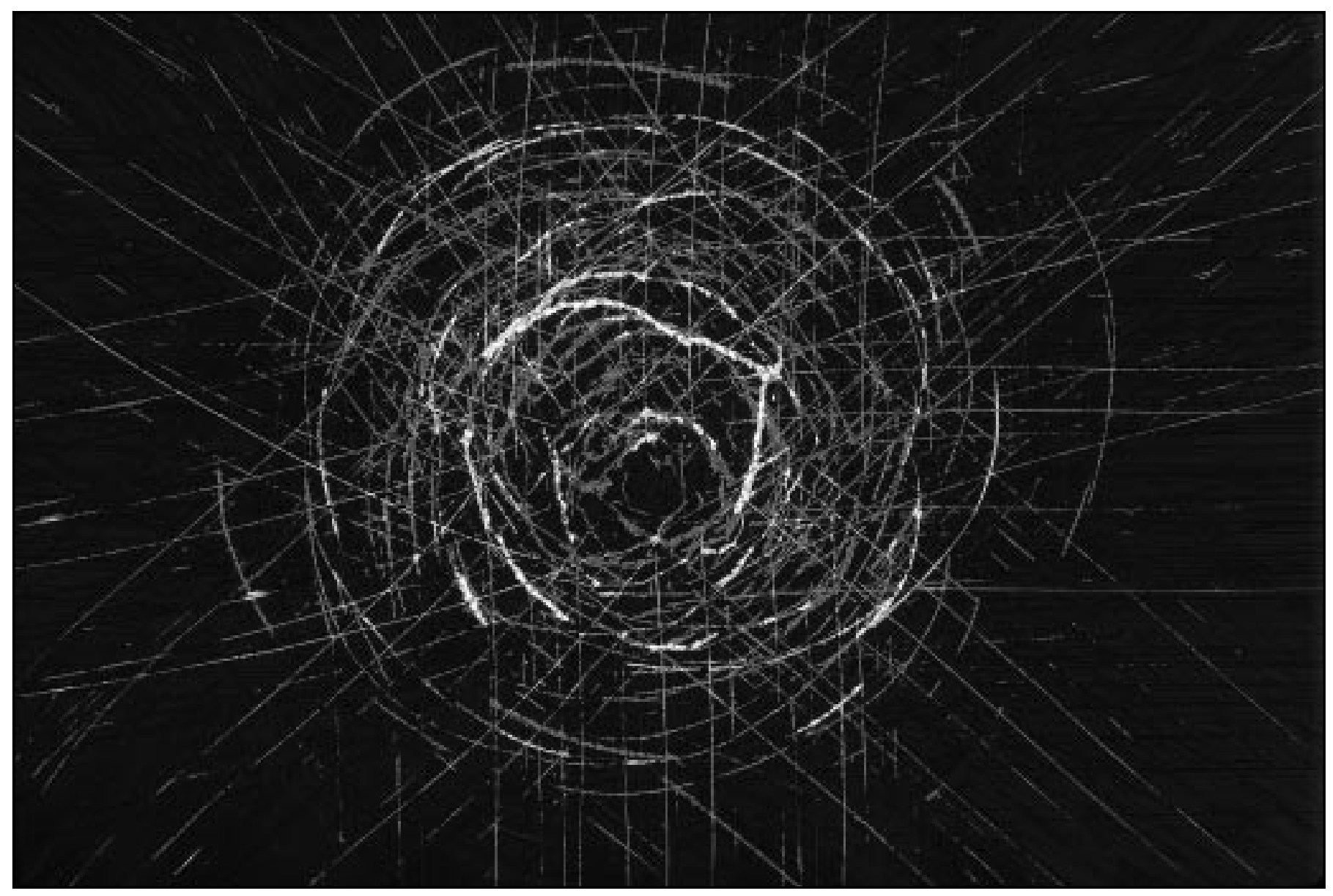

